Research Article

\title{
Lacunary Statistical Convergence in Measure for Double Sequences of Fuzzy Valued Functions
}

\author{
Ömer Kişi 10 \\ Faculty of Science, Department of Mathematics, Bartin University, Bartın, Turkey \\ Correspondence should be addressed to Ömer Kişi; okisi@bartin.edu.tr
}

Received 10 December 2020; Revised 25 January 2021; Accepted 29 January 2021; Published 4 March 2021

Academic Editor: Hijaz Ahmad

Copyright (C) 2021 Ömer Kişi. This is an open access article distributed under the Creative Commons Attribution License, which permits unrestricted use, distribution, and reproduction in any medium, provided the original work is properly cited.

Based on the concept of lacunary statistical convergence of sequences of fuzzy numbers, the lacunary statistical convergence, uniformly lacunary statistical convergence, and equi-lacunary statistical convergence of double sequences of fuzzy-valued functions are defined and investigated in this paper. The relationship among lacunary statistical convergence, uniformly lacunary statistical convergence, equi-lacunary statistical convergence of double sequences of fuzzy-valued functions, and their representations of sequences of $\alpha$-level cuts are discussed. In addition, we obtain the lacunary statistical form of Egorov's theorem for double sequences of fuzzy-valued measurable functions in a finite measurable space. Finally, the lacunary statistical convergence in measure for double sequences of fuzzy-valued measurable functions is examined, and it is proved that the inner and outer lacunary statistical convergence in measure are equivalent in a finite measure set for a double sequence of fuzzy-valued measurable functions.

\section{Introduction}

Fast [1] initiated statistical convergence for a real sequence. After the work of Fridy [2] and Šalát [3], it became a noteworthy topic in summability theory. Mursaleen and Edely [4] examined the statistical convergence via double sequences.

Various types of convergence for sequences of functions, such as pointwise, equi-statistical (or ideal), and uniform convergence, were originated by Balcerzak et al. [5]. Pointwise and uniform statistical convergence of double sequences was studied by Gökhan et al. [6].

Additionally, Duman and Orhan [7] studied convergence in $\mu$-density and $\mu$-statistical convergence of sequences of functions and presented the notions of $\mu$-statistical pointwise convergence and $\mu$-statistical uniform convergence.

Lacunary statistical convergence was firstly studied by Fridy and Orhan [8]. In [9], lacunary statistical convergence via double sequence was investigated.

Zadeh [10] initiated the notion of fuzzy sets. The publication of the paper affected deeply all the scientific fields. This notion is significant for real-life conditions, but has no adequate solution to some problems. Such problems lead to new quests. Matloka [11] identified ordinary convergence of a sequence of fuzzy numbers. Nanda [12] examined the seqeuences with fuzzy numbers. Negoita [13] gave the Hausdorff distance between two fuzzy numbers. Statistical convergence by utilizing fuzzy numbers was given by Nuray and Savaş [14]. Savaş and Mursaleen [15] investigated statistical convergent double sequences via fuzzy numbers. By using fuzzy numbers, Aytar and Pehlivan [16] defined the statistical convergence of sequences. Pointwise statistical convergence sequences of fuzzy mappings studied by Altin et al. [17]. Some beneficial results on this topic can be found in [18-32].

In recent times, Gong et al. studied statistical convergence, equi-statistical convergence, and uniformly statistical convergence for sequences of fuzzy-valued functions. These concepts were extended to the double sequences by Hazarika et al. Kişi and Dündar examined lacunary statistical convergence in measure for sequences of fuzzy-valued functions and established noteworthy results.

According to Zadeh [10], a fuzzy subset of $T$ is a nonempty subset $\{t, \bar{w}(t): t \in T\}$ of $T \times J(=[0,1])$ for some function $\bar{w}: T \longrightarrow J(=[0,1])$. A function $\bar{w}: \mathbb{R} \longrightarrow J(=$ 
$[0,1])$ is called a fuzzy number if the function $\bar{w}$ holds the following properties:

(i) $\bar{w}$ is convex, i.e., $\bar{w}(t) \geq \bar{w}(s) \Lambda \bar{w}(r)=\min \{\bar{w}(s)$, $\bar{w}(r)\}$, where $s<t<r$

(ii) $\bar{w}$ is normal, i.e., there exists an $t_{0} \in \mathbb{R}$ such that $\bar{w}\left(t_{0}\right)=1$

(iii) $\bar{w}$ is upper semicontinuous, i.e., for every $\varepsilon>0$, $\bar{w}^{-1}((0, a+\varepsilon])$, for all $a \in[0,1]$ is open in the usual topology of $\mathbb{R}$

(iv) $[\bar{w}]^{0}=\operatorname{cl}(\{t \in \mathbb{R}: \bar{w}(t)\} \geq 0)$ is compact, where $\mathrm{cl}$ is the closure operator

We indicate the set of all fuzzy numbers by $F(\mathbb{R})$. The set $\mathbb{R}$ of real numbers can be included in $F(\mathbb{R})$ if we take $\bar{r} \in F(\mathbb{R})$ by

$$
\bar{r}(t)= \begin{cases}1, & \text { if } t=r \\ 0, & \text { if } t \neq r\end{cases}
$$

For $0<\alpha \leq 1, \alpha$-cut of $\bar{w}$ is given by $[\bar{w}]_{\alpha}=\{t \in \mathbb{R}$ : $\bar{w}(t) \geq \alpha\}=\left[\bar{w}_{\alpha}^{-}, \bar{w}_{\alpha}^{+}\right]$which is a closed bounded interval of $\mathbb{R}$. As in [13], the Hausdorff distance between two fuzzy numbers $\bar{w}$ and $\bar{q}$ is denoted by $D: F(\mathbb{R}) \times F(\mathbb{R}) \longrightarrow$ $[0, \infty)$ :

$$
\begin{aligned}
D(\bar{w}, \bar{q}) & =\sup _{\alpha \in[0,1]} d\left([\bar{w}]_{\alpha},[\bar{q}]_{\alpha}\right) \\
& =\sup _{\alpha \in[0,1]} \max \left\{\left|\bar{w}_{\alpha}^{-}-\bar{w}_{\alpha}^{+}\right|,\left|\bar{q}_{\alpha}^{-}-\bar{q}_{\alpha}^{+}\right|\right\},
\end{aligned}
$$

where $d$ is the Hausdorff metric.

For $K \subset \mathbb{N}$ and $j \in \mathbb{N}, \delta_{j}(K)$ is named $j$ th partial density of $K$ if

$$
\delta_{j}(K)=\frac{|K \cap\{1,2, \ldots, j\}|}{j} .
$$

If

$$
\delta(K)=\lim _{n \longrightarrow \infty} \frac{1}{n}|\{k \leq n: k \in K\}| \quad\left(\text { i.e., } \delta(K)=\lim _{j \longrightarrow \infty} \delta_{j}(K)\right),
$$

exists, it is named the natural density of $K . \Psi=\{K \subset \mathbb{N}$ : $\delta(K)=0\}$ is denoted the zero density set.

A sequence of fuzzy numbers $\left(\bar{x}_{n}\right)$ is called to be statistically convergent to a fuzzy number $\bar{x}_{0}$ if for every $\varepsilon>0$, $\delta\left(\left\{n \in \mathbb{N}: D\left(\bar{x}_{n}, \bar{x}_{0}\right) \geq \varepsilon\right\}\right)=0$, i.e., $\quad\left\{n \in \mathbb{N}: D\left(\bar{x}_{n}, \bar{x}_{0}\right) \geq \varepsilon\right\}$ $\in \Psi$. It is demonstrated by st $-\lim \bar{x}_{n}=\bar{x}_{0}$ or $\bar{x}_{n} \stackrel{\text { st }}{\longrightarrow} \bar{x}_{0}$, $(n \longrightarrow \infty)$.

Let $A \subset \mathbb{N}$ and $r \in \mathbb{N}$. $\delta_{\theta}^{r}(A)$ is named the $r$ th partial lacunary density of $A$ if

$$
\delta_{\theta}^{r}(A)=\frac{\left|A \cap I_{r}\right|}{h_{r}},
$$

where $I_{r}=\left(k_{r-1}, k_{r}\right]$.

The number $\delta_{\theta}(A)$ is denoted as the lacunary density ( $\theta$-density) of $A$ if

$$
\delta_{\theta}(A)=\lim _{r \longrightarrow \infty} \frac{1}{h_{r}}\left|\left\{k \in I_{r}: k \in A\right\}\right| \quad\left(\text { i.e., } \delta_{\theta}(A)=\lim _{r \longrightarrow \infty} \delta_{\theta}^{r}(A)\right),
$$

exists. Also, $\Lambda=\left\{A \subset \mathbb{N}\right.$ : $\left.\delta_{\theta}(A)=0\right\}$ is called to be zero density set.

A SFVF $\left(\bar{h}_{m}\right)$ is pointwise lacunary statistically convergent to $\operatorname{FVF} \bar{h}$ on $[a, b]$, denoted by $p S_{\theta}-\lim \bar{h}_{m}(z)=$ $\bar{h}(z)$ or $\bar{h}_{m} \longrightarrow^{p} S_{\theta} \bar{h}$; if for each $z \in[a, b]$ and all $\varepsilon>0$ there exists $T_{z} \in \Lambda$ such that, for all $m \in \mathbb{N} \backslash T_{z}$, we get $D\left(\bar{h}_{m}(z), \bar{h}(z)\right)<\varepsilon$. It is obvious that $\bar{h}_{m} \longrightarrow{ }^{p} S_{\theta} \bar{h}$ if for every $z \in[a, b]$ and all $\varepsilon>0, \quad \delta_{\theta}\left(\left\{m \in \mathbb{N}: D\left(\bar{h}_{m}(z)\right.\right.\right.$, $\bar{h}(z)) \geq \varepsilon\})=0$. Here, $\bar{h}$ is named the lacunary statistical limit function of $\left(\bar{h}_{m}\right)$.

Some definitions and significant results about lacunary statistical convergence in measure for sequences of fuzzyvalued functions were given in.

A double sequence $\theta_{2}=\left\{\left(k_{r}, j_{u}\right)\right\}$ is named lacunary sequence if there exist two increasing sequences of integers $\left(k_{u}\right)$ and $\left(j_{u}\right)$ such that

$$
\begin{array}{r}
k_{0}=0, \quad h_{r}=k_{r}-k_{r-1} \longrightarrow \infty, \\
j_{0}=0, \quad \bar{h}_{u}=j_{u}-j_{u-1} \longrightarrow \infty, \\
r, u \longrightarrow \infty .
\end{array}
$$

We use the following symbols in the sequel:

$$
\begin{aligned}
k_{r u} & =k_{r} j_{u}, \\
h_{r u} & =h_{r} \bar{h}_{u}, \\
I_{r u} & =\left\{(k, j): k_{r-1}<k \leq k_{r} \text { and } j_{u-1}<j \leq j_{u}\right\}, \\
q_{r} & =\frac{k_{r}}{k_{r-1}}, \\
\bar{q}_{u} & =\frac{j_{u}}{j_{u-1}}, \\
q_{u r} & =q_{u} \bar{q}_{r} .
\end{aligned}
$$

In the paper, by $\theta_{2}=\left\{\left(k_{r}, j_{u}\right)\right\}$, we will indicate a double lacunary sequence of positive real numbers.

In this article, we proposed the concepts of lacunary statistical convergence, uniformly lacunary statistical convergence, and equi-lacunary statistical convergence for double sequences of fuzzy-valued functions and proved some classical results in this new setting and their representations of sequences of $\alpha$-level cuts. We proved lacunary statistical form of Egorov's theorem for double sequences of fuzzy-valued measurable functions defined on a finite measure space $(\Omega, \mathscr{M}, \mu)$. Finally, we define the notion of lacunary statistical convergence in measure for double sequences of fuzzy-valued measurable functions and prove some interesting results. Our results were emphasized with examples. 


\section{Main Results}

In the section, we presume that $\bar{h}:[a, b] \longrightarrow F(\mathbb{R})$ and $\bar{h}_{m n}:[a, b] \longrightarrow F(\mathbb{R})$ are the fuzzy-valued function and a double sequence of fuzzy-valued functions for all $m, n \in \mathbb{N}$. We indicate FVF and DSFVF in place of fuzzy-valued function and double sequence of fuzzy-valued functions.

Definition 1. Let $T \subset \mathbb{N} \times \mathbb{N}$ and $r, u \in \mathbb{N}$. $\delta_{\theta_{2}}^{r u}(T)$ is called the ruth partial lacunary density of $T$ if

$$
\delta_{\theta_{2}}^{r u}(T)=\frac{\left|T \cap I_{r u}\right|}{h_{r u}} .
$$

The number $\delta_{\theta_{2}}(T)$ is named the lacunary density or $\theta_{2}$-density of $T$ if

$$
\begin{gathered}
\delta_{\theta_{2}}(T)=\lim _{r, u \longrightarrow \infty} \frac{1}{h_{r u}}\left|\left\{(k, j) \in I_{r u}:(k, j) \in T\right\}\right| \\
\text {. (i.e., } \left.\delta_{\theta_{2}}(T)=\lim _{r, u \longrightarrow \infty} \delta_{\theta_{2}}^{r u}(T)\right)
\end{gathered}
$$

exists. Additionally,

$$
\Lambda_{2}=\left\{T \subset \mathbb{N} \times \mathbb{N}: \delta_{\theta_{2}}(T)=0\right\}
$$

is named to be zero density set.

Definition 2. We call that a DSFVF $\left(\bar{h}_{m n}\right)$ is pointwise lacunary statistically convergent to $\operatorname{FVF} \bar{h}$ on $[a, b]$, indicated by

$$
p S_{\theta_{2}}-\lim \bar{h}_{m n}(z)=\bar{h}(z) \text { or } \bar{h}_{m n} \stackrel{p}{\longrightarrow} S_{\theta_{2}} \bar{h},
$$

if for every $z \in[a, b]$ and for all $\varepsilon>0$ there exists $T_{z} \in \Lambda_{2}$ such that, for all $(m, n) \in \mathbb{N} \times \mathbb{N} \backslash T_{z}$, we have

$$
D\left(\bar{h}_{m n}(z), \bar{h}(z)\right)<\varepsilon \text {. }
$$

It is obvious that $\bar{h}_{m n} \longrightarrow^{p} S_{\theta_{2}} \bar{h}$ if for every $z \in[a, b]$ and for each $\varepsilon>0$

$$
\delta_{\theta_{2}}\left(\left\{(m, n) \in \mathbb{N} \times \mathbb{N}: D\left(\bar{h}_{m n}(z), \bar{h}(z)\right) \geq \varepsilon\right\}\right)=0 .
$$

Definition 3. We call that a DSFVF $\left(\bar{h}_{m n}\right)$ is uniformly lacunary statistically convergent to FVF $\bar{h}$ on $[a, b]$, denoted by $u S_{\theta_{2}}-\lim \bar{h}_{m n}(z)=\bar{h}(z)$ on $[a, b]$, or $\bar{h}_{m n} \stackrel{u S_{\theta_{2}}}{\rightrightarrows} \bar{h}$, if for each $\varepsilon>0$ there exists $T \in \Lambda_{2}$ such that, for all $(m, n) \in \mathbb{N} \times \mathbb{N} \backslash T$, we obtain

$$
D\left(\bar{h}_{m n}(z), \bar{h}(z)\right)<\varepsilon,
$$

which provides for all $z \in[a, b]$. It is obvious that $\bar{h}_{m n} \stackrel{u S_{\theta_{2}}}{\rightrightarrows} \bar{h}$ if, for all $\varepsilon>0$,

$$
\delta_{\theta_{2}}\left(\left\{(m, n) \in \mathbb{N} \times \mathbb{N}: D\left(\bar{h}_{m n}(z), \bar{h}(z)\right) \geq \varepsilon\right\}\right)=0,
$$

for all $z \in[a, b]$.

Remark 1. If $\bar{h}_{m n} \stackrel{u S_{\theta_{2}}}{\rightrightarrows} \bar{h}$, then $\bar{h}_{m n} \longrightarrow p S_{\theta_{2}} \bar{h}$.
Remark 2. $\bar{h}_{m n} \stackrel{u S_{\theta_{2}}}{\rightrightarrows} \bar{h}$ iff $\sup _{z \in[a, b]} D\left(\bar{h}_{m n}(z), \bar{h}(z)\right) \longrightarrow p S_{\theta_{2}} 0$.

Proof. Assume that $\sup _{z \in[a, b]} D\left(\bar{h}_{m n}(z), \bar{h}(z)\right) \stackrel{p s_{\theta_{2}}}{\longrightarrow} 0$. Then, for each $z \in[a, b]$ and for every $\varepsilon>0$, we have $D\left(\bar{h}_{m n}(z), \bar{h}(z)\right) \leq \sup _{z \in[a, b]} D\left(\bar{h}_{m n}(z), \bar{h}(z)\right)$ so that

$\left\{(m, n) \in \mathbb{N} \times \mathbb{N}: D\left(\bar{h}_{m n}(z), \bar{h}(z)\right) \geq \varepsilon, \forall z \in[a, b]\right\}$

$$
\subseteq\left\{(m, n) \in \mathbb{N} \times \mathbb{N}: \sup _{z \in[a, b]} D\left(\bar{h}_{m n}(z), \bar{h}(z)\right) \geq \varepsilon\right\} .
$$

Hence, $\bar{h}_{m n} \stackrel{u s_{\theta_{2}}}{\rightrightarrows} \bar{h}$.

Next, suppose that $\bar{h}_{m n} \stackrel{u S_{\theta_{2}}}{\rightrightarrows} \bar{h}$ on $[a, b]$. For every $\varepsilon>0$, we write

$$
\begin{aligned}
& A_{1}=\left\{(m, n) \in \mathbb{N} \times \mathbb{N}: D\left(\bar{h}_{m n}(z), \bar{h}(z)\right) \geq \varepsilon, \forall z \in[a, b]\right\}, \\
& A_{2}=\left\{(m, n) \in \mathbb{N} \times \mathbb{N}: \sup _{z \in[a, b]} D\left(\bar{h}_{m n}(z), \bar{h}(z)\right) \geq \varepsilon\right\} .
\end{aligned}
$$

If $(m, n) \in \mathbb{N} \times \mathbb{N} \backslash A_{1}$, then $D\left(\bar{h}_{m n}(z), \bar{h}(z)\right)<\varepsilon, \forall z \in[a$, b] which implies

$$
\sup _{z \in[a, b]} D\left(\bar{h}_{m n}(z), \bar{h}(z)\right)<\varepsilon,
$$

as $\varepsilon$ is arbitrary. This gives that $(m, n) \in \mathbb{N} \times \mathbb{N} \backslash A_{2}$. Therefore, we obtain

$$
\mathbb{N} \times \mathbb{N} \backslash A_{1} \subseteq \mathbb{N} \times \mathbb{N} \backslash A_{2} \Rightarrow A_{2} \subseteq A_{1},
$$

which gives $\delta_{\theta_{2}}\left(A_{2}\right) \leq \delta_{\theta_{2}}\left(A_{1}\right)=0$. Hence, $\sup _{z \in[a, b]} D\left(\bar{h}_{m n}(z), \bar{h}(z)\right) \stackrel{2}{\longrightarrow} S_{\theta_{2}} 0$.

Theorem 1. Presume that the sequence of FVF $\bar{h}_{m n}$ $\longrightarrow^{p} S_{\theta_{2}} \bar{h}$ on $[a, b]$, where $\left(\bar{h}_{m n}\right)$ is equi-continuous on $[a, b]$; then, $\bar{h}$ is continuous and $\bar{h}_{m n} \stackrel{u S_{\theta_{2}}}{\rightrightarrows} \bar{h}$ on $[a, b]$.

Proof. First, we demonstrate that $\bar{h}_{m n}$ is continuous. Let $\varepsilon>0$ and $z_{0} \in[a, b]$. By the equi-continuity of $\bar{h}_{m n}$, then there exists $\gamma>0$ such that

$$
D\left(\bar{h}_{m n}(z), \bar{h}_{m n}\left(z_{0}\right)\right)<\frac{\varepsilon}{3},
$$

for any $(m, n) \in \mathbb{N} \times \mathbb{N}$ and $z \in\left(z_{0}-\gamma, z_{0}+\gamma\right)$. For some $z \in\left(z_{0}-\gamma, z_{0}+\gamma\right)$, since $\bar{h}_{m n} \longrightarrow^{p} S_{\theta_{2}} \bar{h}$, the set

$$
\begin{aligned}
& \left\{(m, n) \in \mathbb{N} \times \mathbb{N}: D\left(\bar{h}_{m n}\left(z_{0}\right), \bar{h}\left(z_{0}\right)\right) \geq \frac{\varepsilon}{3}\right\} \\
& \quad \cup\left\{(m, n) \in \mathbb{N} \times \mathbb{N}: D\left(\bar{h}_{m n}(z), \bar{h}(z)\right) \geq \frac{\varepsilon}{3}\right\} \in \Lambda_{2} .
\end{aligned}
$$

So, there exists $(m, n) \in \mathbb{N} \times \mathbb{N}$ such that

$$
\begin{aligned}
D\left(\bar{h}_{m n}\left(z_{0}\right), \bar{h}\left(z_{0}\right)\right) & <\frac{\varepsilon}{3}, \\
D\left(\bar{h}_{m n}(z), \bar{h}(z)\right) & <\frac{\varepsilon}{3} .
\end{aligned}
$$

We obtain 


$$
\begin{aligned}
D\left(\bar{h}\left(z_{0}\right), \bar{h}(z)\right) \leq & D\left(\bar{h}\left(z_{0}\right), \bar{h}_{m n}\left(z_{0}\right)\right)+D\left(\bar{h}_{m n}\left(z_{0}\right), \bar{h}_{m n}(z)\right) \\
& +D\left(\bar{h}_{m n}(z), \bar{h}(z)\right)<\frac{\varepsilon}{3}+\frac{\varepsilon}{3}+\frac{\varepsilon}{3}=\varepsilon .
\end{aligned}
$$

Therefore, we proved the continuity of $\bar{h}$.

At the moment, we will show that $\bar{h}_{m n} \stackrel{u S_{\theta_{2}}}{\rightrightarrows} \bar{h}$ on $[a, b]$. Let $\varepsilon>0$. Since $\bar{h}$ is continuous on $[a, b]$, it provides that $\bar{h}$ is uniformly continuous and $\left(\bar{h}_{m n}\right)$ is equi-uniformly continuous on $[a, b]$. So, pick $\gamma>0$ such that, for any $z, z^{\prime} \in[a$, b] and $\left|z-z^{\prime}\right|<\gamma$, we have

$$
\begin{aligned}
D\left(\bar{h}_{m n}(z), \bar{h}_{m n}\left(z^{\prime}\right)\right) & <\frac{\varepsilon}{3}, \\
D\left(\bar{h}(z), \bar{h}\left(z^{\prime}\right)\right) & <\frac{\varepsilon}{3} .
\end{aligned}
$$

Using the finite covering theorem, select finite open coverings

$$
\left(z_{1}-\gamma, z_{1}+\gamma\right),\left(z_{2}-\gamma, z_{2}+\gamma\right), \ldots,\left(z_{r}-\gamma, z_{r}+\gamma\right),
$$

from the cover of $[a, b]$. Using $\bar{h}_{m n} \longrightarrow p S_{\theta_{2}} \bar{h}$, there exists a set $M_{z_{i}} \in \Lambda_{2}$ such that

$$
D\left(\bar{h}_{m n}\left(z_{i}\right), \bar{h}\left(z_{i}\right)\right)<\frac{\varepsilon}{3},
$$

for all $(m, n) \notin M_{z_{i}}$ and $i \in\{(1,1),(2,2) \ldots,(r, u)\}$. Let $(m, n) \notin M_{z_{i}}$ and $z \in[a, b]$. Hence, $z \in\left(z_{i}-\gamma, z_{i}+\gamma\right)$ for some $i \in\{(1,1),(2,2) \ldots,(r, u)\}$. As a result

$$
\begin{aligned}
D\left(\bar{h}_{m n}(z), \bar{h}(z)\right) \leq & D\left(\bar{h}_{m n}(z), \bar{h}_{m n}\left(z_{i}\right)\right)+D\left(\bar{h}_{m n}\left(z_{i}\right), \bar{h}\left(z_{i}\right)\right) \\
& +D\left(\bar{h}\left(z_{i}\right), \bar{h}(z)\right)<\frac{\varepsilon}{3}+\frac{\varepsilon}{3}+\frac{\varepsilon}{3}=\varepsilon,
\end{aligned}
$$

which gives that $\bar{h}_{m n} \stackrel{u S_{\theta_{2}}}{\rightrightarrows} \bar{h}$ on $[a, b]$.

Definition 4. We call that a DSFVF $\left(\bar{h}_{m n}\right)$ is equi-lacunary statistically convergent to FVF $\bar{h}$, denoted by $\bar{h}_{m n} \longrightarrow S_{\theta_{2}} \bar{h}$, if, for given $\varepsilon>0$,

$$
G_{r u, \varepsilon}=\delta_{\theta_{2}}^{r u}\left(\left\{(m, n) \in \mathbb{N} \times \mathbb{N}: D\left(\bar{h}_{m n}(z), \bar{h}(z)\right) \geq \varepsilon\right\}\right),
$$

with regards to $z \in[a, b]$ is uniformly convergent to zero function. Thus, $\bar{h}_{m n} \longrightarrow{ }^{e} S_{\theta_{2}} \bar{h}$ iff for all $\varepsilon, \beta>0, \exists k, l \in \mathbb{N}$, for each $r \geq k, u \geq l$, for all $z \in[a, b]$,

$$
\delta_{\theta_{2}}^{r u}\left(\left\{(m, n) \in \mathbb{N} \times \mathbb{N}: D\left(\bar{h}_{m n}(z), \bar{h}(z)\right) \geq \varepsilon\right\}\right)<\beta .
$$
$\beta=\varepsilon$.

Note that, by monotonicity of $\delta_{\theta_{2}}^{r u}$, we can also utilize

Remark 3. It is obvious that $\bar{h}_{m n} \longrightarrow p S_{\theta_{2}} \bar{h}$ iff for every $z \in Y$ and for every $\varepsilon, \beta>0 \exists k, l \in \mathbb{N}$, for all $r \geq k, u \geq l$,

$$
\delta_{\theta_{2}}^{r u}\left(\left\{(m, n) \in \mathbb{N} \times \mathbb{N}: D\left(\bar{h}_{m n}(z), \bar{h}(z)\right) \geq \varepsilon\right\}\right)<\beta .
$$

In this instance, we may take $\beta=\varepsilon$. Obviously, $\bar{h}_{m n} \longrightarrow^{e} S_{\theta_{2}} \bar{h}$ implies $\bar{h}_{m n} \longrightarrow{ }^{p} S_{\theta_{2}} \bar{h}$. Furthermore, we see that $\bar{h}_{m n} \stackrel{u S_{\theta_{2}}}{\rightrightarrows} \bar{h}$ indicates $\bar{h}_{m n} \longrightarrow S_{\theta_{2}} \bar{h}$.

Corollary 1. Let $\left(\bar{h}_{m n}\right)$ be a DSFVF and $\bar{h}$ be a FVF on $[a, b]$. Then, $\bar{h}_{m n} \stackrel{u S_{\theta_{2}}}{\rightrightarrows} \bar{h} \Rightarrow \bar{h}_{m n} \longrightarrow^{e} S_{\theta_{2}} \bar{h} \Rightarrow \bar{h}_{m n} \longrightarrow p S_{\theta_{2}} \bar{h}$. The converse implications do not hold in general.

Proof. Proving the above result, we can consider the examples as given below.

Example 1. Consider DSFVF $\left(\bar{h}_{m n}\right)$ is defined by $\bar{h}_{m n}(z)=$ $\overline{\left(e^{-m n z}\right)}$ for $z \in[0,1]$. Then, we get $D\left(\bar{h}_{m n}(z), \overline{0}\right)=e^{-m n z}$ for each $(m, n) \in \mathbb{N} \times \mathbb{N}$ and $z \in[0,1]$. Therefore, $\left(\bar{h}_{m n}\right)$ converges pointwise to $\bar{h}=\overline{0}$ and so $\bar{h}_{m n} \longrightarrow^{p} S_{\theta_{2}} \overline{0}$. However, for each $(k, l) \in \mathbb{N} \times \mathbb{N}$, we consider $(k, l) \in[m n, 2 m n-1]$. Therefore, for all $z \in[0,1 / 2 m n-1]$, we have

$$
\begin{aligned}
D\left(\bar{h}_{k l}(z), \overline{0}\right) & =e^{-m n z} \\
& \geq e^{-(2 m n-1) z} \\
& \geq \frac{1}{e}\left(\operatorname{as} z \in\left[0, \frac{1}{2 m n-1}\right]\right) \\
& \geq \frac{1}{3} .
\end{aligned}
$$

Thus, for all $z \in[0,1 /(2 m n-1)]$, one obtains

$$
\begin{aligned}
A_{m n}(z)= & \delta_{\theta_{2}}\left(\left\{(k, l) \in[m n, 2 m n-1]: D\left(\bar{h}_{k l}(z), \overline{0}\right) \geq \frac{1}{3}\right\}\right) \\
& \longrightarrow 1(\neq 0) .
\end{aligned}
$$

Hence, $\left(\bar{h}_{m n}\right)$ is not equi-lacunary statistically convergent to $\overline{0}$ on $[0,1]$.

Example 2. Let us define a DSFVF $\left(\bar{h}_{m n}\right)$ by

$$
\bar{h}_{m n}(z)= \begin{cases}\overline{\left(\frac{m^{2} n^{2} z}{1+m^{3} n^{3} z^{2}}\right)}, & \text { for } z \in\left[\frac{1}{m n+2}, \frac{1}{m n+1}\right], \\ \overline{0}, & \text { otherwise. }\end{cases}
$$

Then, for every $z \in[0,1]$, we have

$$
\delta_{\theta_{2}}\left(\left\{(m, n) \in \mathbb{N} \times \mathbb{N}: \bar{h}_{m n}(z) \neq \overline{0}\right\}\right)=0 .
$$

Therefore, for every $\varepsilon>0$, 


$$
\begin{aligned}
& \frac{1}{h_{r u}}\left|\left\{(m, n) \in I_{r u}: D\left(\bar{h}_{m n}(z), \overline{0}\right) \geq \varepsilon\right\}\right| \\
& \quad \leq \frac{1}{h_{r u}}\left|\left\{(m, n) \in I_{r u}: \bar{h}_{m n}(z) \neq \overline{0}\right\}\right| \\
& \cap\{(1,1),(2,2), \ldots,(m, n)\} \leq \frac{1}{h_{r u}} \longrightarrow 0 .
\end{aligned}
$$

This yields that $\left(\bar{h}_{m n}\right)$ is pointwise lacunary statistically convergent to $\bar{h}=\overline{0}$. However,

$$
\begin{aligned}
\sup _{z \in[0,1]} D\left(\bar{h}_{m n}(z), \overline{0}\right) & =\sup _{z \in[0,1]} \frac{m^{2} n^{2} z}{1+m^{3} n^{3} z^{2}} \\
& =\sup _{z \in[0,1]} \frac{1}{\left(1 / m^{2} n^{2} z\right)+m n z} \neq 0,
\end{aligned}
$$

for all $m, n \in \mathbb{N}$ and $\left(\bar{h}_{m n}\right)$ is not uniformly lacunary statistically convergent to $\bar{h}=\overline{0}$ on $[0,1]$.

Theorem 2. A of SFVMF $\left(\bar{h}_{m n}\right)$ is uniformly lacunary statistically convergent to FVMF $\bar{h}$ iff $\left[\bar{h}_{m n}(z)\right]_{\alpha}$ is uniformly lacunary statistically convergent to $[\bar{h}(z)]_{\alpha}$ uniformly with regards to $\alpha$ and $z$.

Proof. Select $\varepsilon>0$. Given $\bar{h}_{m n} \stackrel{u S_{\theta_{2}}}{\rightrightarrows} \bar{h}$, there exists $M \in \Lambda_{2}$ such that $D\left(\bar{h}_{m n}(z), \bar{h}(z)\right)<\varepsilon$, for any $(m, n) \in \mathbb{N} \times \mathbb{N} \backslash M$ and $z \in[a, b]$, i.e.,

$$
\sup _{\alpha \in[0,1]} \max \left\{\left|\left(\bar{h}_{m n}(z)\right)_{\alpha}^{-}-\bar{h}_{\alpha}^{-}(z)\right|,\left|\left(\bar{h}_{m n}(z)\right)_{\alpha}^{+}-\bar{h}_{\alpha}^{+}(z)\right|\right\}<\varepsilon .
$$

That is, there are

$$
\begin{aligned}
& \left|\left(\bar{h}_{m n}(z)\right)_{\alpha}^{-}-\bar{h}_{\alpha}^{-}(z)\right|<\varepsilon, \\
& \left|\left(\bar{h}_{m n}(z)\right)_{\alpha}^{+}-\bar{h}_{\alpha}^{+}(z)\right|<\varepsilon,
\end{aligned}
$$

for any $(m, n) \in \mathbb{N} \times \mathbb{N} \backslash M$ and $z \in[a, b]$. Moreover,

$$
\begin{aligned}
{\left[\bar{h}_{m n}(z)\right]_{\alpha} } & =\left[\left(\bar{h}_{m n}(z)\right)_{\alpha}^{-},\left(\bar{h}_{m n}(z)\right)_{\alpha}^{+}\right], \\
{[\bar{h}(z)]_{\alpha} } & =\left[\bar{h}_{\alpha}^{-}(z), \bar{h}_{\alpha}^{+}(z)\right] .
\end{aligned}
$$

Consequently, we get $\left[\bar{h}_{m n}(z)\right]_{\alpha}$ is uniformly lacunary statistically convergent to $[\bar{h}(z)]_{\alpha}$ uniformly with regards to $\alpha$ and $z$.

Conversely, for any $\alpha \in[0,1]$ and for any $z \in[a, b]$, $\left[\bar{h}_{m n}(z)\right]_{\alpha}$ is uniformly lacunary statistically convergent to $[\bar{h}(z)]_{\alpha}$ with regards to $\alpha$ and $z$. Thus, for given $\varepsilon>0$, there exists $M_{1} \in \Lambda_{2}$ such that

$$
\left|\left(\bar{h}_{m n}(z)\right)_{\alpha}^{-}-\bar{h}_{\alpha}^{-}(z)\right|<\varepsilon,
$$

for all $(m, n) \in \mathbb{N} \times \mathbb{N} \backslash M_{1}, z \in[a, b]$, and any $\alpha \in[0,1]$. Additionally, we see that, for given $\varepsilon>0$, there exists $M_{2} \in \Lambda_{2}$ such that

$$
\left|\left(\bar{h}_{m n}(z)\right)_{\alpha}^{+}-\bar{h}_{\alpha}^{+}(z)\right|<\varepsilon,
$$

for all $(m, n) \in \mathbb{N} \times \mathbb{N} \backslash M_{2}, z \in[a, b]$ and any $\alpha \in[0,1]$. Take $M=M_{1} \cup M_{2} \in \Lambda_{2}$. We get

$$
\begin{gathered}
\left|\left(\bar{h}_{m n}(z)\right)_{\alpha}^{-}-\bar{h}_{\alpha}^{-}(z)\right|<\varepsilon, \\
\left|\left(\bar{h}_{m}(z)\right)_{\alpha}^{+}-\bar{h}_{\alpha}^{+}(z)\right|<\varepsilon,
\end{gathered}
$$

for all $(m, n) \in \mathbb{N} \times \mathbb{N} \backslash M, z \in[a, b]$, and any $\alpha \in[0,1]$. As a result, we have

$$
\sup _{\alpha \in[0,1]} \max \left\{\left|\left(\bar{h}_{m n}(z)\right)_{\alpha}^{-}-\bar{h}_{\alpha}^{-}(z)\right|,\left|\left(\bar{h}_{m n}(z)\right)_{\alpha}^{+}-\bar{h}_{\alpha}^{+}(z)\right|\right\}<\varepsilon,
$$

that is,

$$
D\left(\bar{h}_{m n}(z), \bar{h}(z)\right)<\varepsilon .
$$

This concludes the proof.

Theorem 3. Let $\bar{h}$ FVMF and $\left(\bar{h}_{m n}\right)$ SFVMF. Fix $z_{0} \in[a, b]$ if $\bar{h}_{m n} \longrightarrow S_{\theta_{2}} \bar{h}$ on $[a, b]$ and all $\left(\bar{h}_{m n}\right)$ are continuous on $z_{0}$, then $\bar{h}$ is continuous on $z_{0}$.

Proof. Let $\varepsilon>0 . \bar{h}_{m n} \longrightarrow^{e} S_{\theta_{2}} \bar{h}$, and we can identify numbers $r, u \in \mathbb{N}$ such that, for all $z \in[a, b]$,

$$
\delta_{\theta_{2}}^{r u}\left(\left\{(m, n) \in \mathbb{N} \times \mathbb{N}: D\left(\bar{h}_{m n}(z), \bar{h}(z)\right) \geq \frac{\varepsilon}{3}\right\}\right)<\frac{1}{2} .
$$

Let $K(z)=\left\{(m, n) \in \mathbb{N} \times \mathbb{N}: D\left(\bar{h}_{m n}(z), \bar{h}(z)\right)<(\varepsilon / 3)\right\}$, $z \in[a, b]$. Therefore, $\delta_{\theta_{2}}^{r u}(K(z))>(1 / 2)$ for all $z \in[a, b]$. Via the continuity of $\bar{h}_{11}, \bar{h}_{22}, \ldots, \bar{h}_{r u}$ at $z_{0}$, there is a neighborhood $\left(z_{0}-\zeta, z_{0}+\zeta\right)$ of $z_{0}$ such that

$$
D\left(\bar{h}_{i}(z), \bar{h}_{i}\left(z_{0}\right)\right)<\frac{\varepsilon}{3},
$$

for all $i=\{(1,1),(2,2) \ldots,(r, u)\}$ and $z \in\left(z_{0}-\zeta, z_{0}+\zeta\right)$. Fix $z \in\left(z_{0}-\zeta, z_{0}+\zeta\right)$. Since $\delta_{\theta_{2}}^{r u}(K(z))>(1 / 2)$ and $\delta_{\theta_{2}}^{r u}\left(K\left(z_{0}\right)\right)>(1 / 2)$, we find $p \in K(z)^{2} \cap K\left(z_{0}\right)$. Thus,

$$
\begin{aligned}
D\left(\bar{h}(z), \bar{h}\left(z_{0}\right)\right) \leq & D\left(\bar{h}(z), \bar{h}_{p}(z)\right)+D\left(\bar{h}_{p}(z), \bar{h}_{p}\left(z_{0}\right)\right) \\
& +D\left(\bar{h}_{p}\left(z_{0}\right), \bar{h}\left(z_{0}\right)\right)<\frac{\varepsilon}{3}+\frac{\varepsilon}{3}+\frac{\varepsilon}{3}=\varepsilon .
\end{aligned}
$$

Thus, we obtain

$$
D\left(\bar{h}(z), \bar{h}\left(z_{0}\right)\right)<\varepsilon,
$$

for all $z \in \mathscr{U}\left(z_{0}, \zeta\right)$, i.e., $\bar{h}$ is continuous on $z_{0}$.

Theorem 4. A of SFVMF $\left(\bar{h}_{m n}\right)$ is equi-lacunary statistically convergent to FVMF $\bar{h}$ iff $\left[\bar{h}_{m n}(z)\right]_{\alpha}$ is equi-lacunary statistically convergent to $[\bar{h}(z)]_{\alpha}$ uniformly for any $\alpha \in[0,1]$ and any $z \in[a, b]$. 
Proof. $\bar{h}_{m n} \longrightarrow^{e} S_{\theta} \bar{h}$ shows that, for any $\varepsilon>0$ and $\sigma>0$, there exists $k, l \in \mathbb{N}$, for all $r \geq k, u \geq l$ and any $z \in[a, b]$ such that $\delta_{\theta_{2}}^{r u}\left(\left\{(m, n) \in \mathbb{N} \times \mathbb{N}: D\left(\bar{h}_{m n}(z), \bar{h}(z)\right) \geq \varepsilon\right\}\right)<\sigma$.

So, for any $\alpha \in[0,1]$, we obtain

$$
\delta_{\theta_{2}}^{r u}\left(\left\{(m, n) \in \mathbb{N} \times \mathbb{N}: \sup _{\alpha \in[0,1]} \max \left\{\left|\left(\bar{h}_{m n}(z)\right)_{\alpha}^{-}-\bar{h}_{\alpha}^{-}(z)\right|,\left|\left(\bar{h}_{m n}(z)\right)_{\alpha}^{+}-\bar{h}_{\alpha}^{+}(z)\right|\right\} \geq \varepsilon\right\}\right)<\sigma .
$$

Therefore, for any $\alpha \in[0,1]$, we acquire

$$
\begin{aligned}
& \delta_{\theta_{2}}^{r u}\left(\left\{(m, n) \in \mathbb{N} \times \mathbb{N}:\left|\left(\bar{h}_{m n}(z)\right)_{\alpha}^{-}-\bar{h}_{\alpha}^{-}(z)\right| \geq \varepsilon\right\}\right)<\sigma, \\
& \delta_{\theta_{2}}^{r u}\left(\left\{(m, n) \in \mathbb{N} \times \mathbb{N}:\left|\left(\bar{h}_{m n}(z)\right)_{\alpha}^{+}-\bar{h}_{\alpha}^{+}(z)\right| \geq \varepsilon\right\}\right)<\sigma .
\end{aligned}
$$

Then, emphasize that

$$
\begin{aligned}
{\left[\bar{h}_{m n}(z)\right]_{\alpha} } & =\left[\left(\bar{h}_{m n}(z)\right)_{\alpha}^{-},\left(\bar{h}_{m n}(z)\right)_{\alpha}^{+}\right], \\
{[\bar{h}(z)]_{\alpha} } & =\left[\bar{h}_{\alpha}^{-}(z), \bar{h}_{\alpha}^{+}(z)\right] .
\end{aligned}
$$

Hence, $\left[\bar{h}_{m n}(z)\right]_{\alpha}$ is uniformly lacunary statistically convergent to $[\bar{h}(z)]_{\alpha}$ for any $\alpha \in[0,1]$ and any $z \in[a, b]$.

Conversely, let $\varepsilon>0$ and $\sigma>0$, and there exists $k_{1}, l_{1} \in \mathbb{N}$ such that

$$
\delta_{\theta_{2}}^{r u}\left(\left\{(m, n) \in \mathbb{N} \times \mathbb{N}:\left|\left(\bar{h}_{m n}(z)\right)_{\alpha}^{-}-\bar{h}_{\alpha}^{-}(z)\right| \geq \varepsilon\right\}\right)<\sigma,
$$

for all $r \geq k_{1}, u \geq l_{1}$ and any $z \in[a, b]$ and for any $\alpha \in[0,1]$. Similarly, there exists $k_{2}, l_{2} \in \mathbb{N}$ such that

$$
\delta_{\theta_{2}}^{r u}\left(\left\{(m, n) \in \mathbb{N} \times \mathbb{N}:\left|\left(\bar{h}_{m n}(z)\right)_{\alpha}^{+}-\bar{h}_{\alpha}^{+}(z)\right| \geq \varepsilon\right\}\right)<\sigma,
$$

for all $r \geq k_{2}$ and $u \geq l_{2}$ and any $z \in[a, b]$ and for any $\alpha \in[0,1]$. Then, select $k=\max \left\{k_{1}, k_{2}\right\}$ and $l=\max \left\{l_{1}, l_{2}\right\}$. We obtain

$$
\begin{aligned}
& \delta_{\theta_{2}}^{r u}\left(\left\{(m, n) \in \mathbb{N} \times \mathbb{N}:\left|\left(\bar{h}_{m n}(z)\right)_{\alpha}^{-}-\bar{h}_{\alpha}^{-}(z)\right| \geq \varepsilon\right\}\right)<\sigma, \\
& \delta_{\theta_{2}}^{r u}\left(\left\{(m, n) \in \mathbb{N} \times \mathbb{N}:\left|\left(\bar{h}_{m n}(z)\right)_{\alpha}^{+}-\bar{h}_{\alpha}^{+}(z)\right| \geq \varepsilon\right\}\right)<\sigma,
\end{aligned}
$$

for all $r \geq k$ and $u \geq l$ and any $z \in[a, b]$ and for any $\alpha \in[0,1]$. Thus, we have

$$
\delta_{\theta_{2}}^{r u}\left(\left\{(m, n) \in \mathbb{N} \times \mathbb{N}: \sup _{\alpha \in[0,1]} \max \left\{\left|\left(\bar{h}_{m n}(z)\right)_{\alpha}^{-}-\bar{h}_{\alpha}^{-}(z)\right|,\left|\left(\bar{h}_{m n}(z)\right)_{\alpha}^{+}-\bar{h}_{\alpha}^{+}(z)\right|\right\} \geq \varepsilon\right\}\right)<\sigma
$$

that is,

$$
\delta_{\theta_{2}}^{r u}\left(\left\{(m, n) \in \mathbb{N} \times \mathbb{N}: D\left(\bar{h}_{m n}(z), \bar{h}(z)\right) \geq \varepsilon\right\}\right)<\sigma .
$$

This concludes the proof.

The next result is the lacunary statistical form of Egorov's theorem for the DSFVF.

Theorem 5. Take measurable space $(\Omega, \mathscr{M}, \mu)$. Presume that $\bar{h}$ and $\left(\bar{h}_{m n}\right)$ are measurable and identified almost everywhere on $\Omega$. Suppose also that $\bar{h}_{m n} \longrightarrow{ }^{p} S_{\theta_{2}} \bar{h}$ almost everywhere on $\Omega$. Then, for every $\varepsilon>0$, there exists $\mathscr{A} \subset M$ such that $\mu(\Omega \backslash \mathscr{A})<\varepsilon$ and $\bar{h}_{m n \mid \mathscr{A}} \longrightarrow^{e} S_{\theta_{2}} \bar{h}_{\mid \mathscr{A}}$ on $\mathscr{A}$.

Proof. We presume that $\bar{h}$ and $\left(\bar{h}_{m n}\right)$ are defined everywhere on $\Omega$ and also suppose that $\bar{h}_{m n}(z) \longrightarrow^{p} S_{\theta_{2}} \bar{h}(z)$ for all $z \in \Omega$. Now, for any fix $\sigma, r, u \in \mathbb{N}$, consider that the set

$$
P=\left\{z \in \Omega: \delta_{\theta_{2}}^{r u}\left(\left\{(m, n) \in \mathbb{N} \times \mathbb{N}: D\left(\bar{h}_{m n}(z), \bar{h}(z)\right) \geq \frac{1}{\sigma}\right\}\right)<\frac{1}{\sigma}\right\},
$$

is measurable. Then, the function $\Phi_{m n}(z)=D\left(\bar{h}_{m n}(z)\right.$, $\bar{h}(z)), z \in \Omega$, is measurable. Let $H_{m n}=\Phi_{m n}^{-1}([(1 / \sigma), \infty))$. For every $z \in \Omega$, we have $z \in P$ iff

$$
\frac{1}{h_{r u}} \sum_{m=1}^{r} \sum_{n=1}^{u} \chi_{H_{m n}}(z)<\frac{1}{\sigma} .
$$

Since the function

$$
h=\frac{1}{h_{r u}} \sum_{m=1}^{r} \sum_{n=1}^{u} \chi_{H_{m n}}(z)
$$

is measurable, so we have $P=h^{-1}((-\infty,(1 / \sigma)))$. For $k, l \in \mathbb{N}$, one writes

$$
\begin{aligned}
\Psi_{\sigma, k, l}= & \left\{z \in \Omega: \forall r \geq k, u \geq l, \delta_{\theta_{2}}^{r u}\right. \\
& \left.\left(\left\{(m, n) \in \mathbb{N} \times \mathbb{N}: D\left(\bar{h}_{m n}(z), \bar{h}(z)\right) \geq \frac{1}{\sigma}\right\}\right)<\frac{1}{\sigma}\right\} .
\end{aligned}
$$

From the previous observation, we conclude that $\Psi_{\sigma, k, l}$ is measurable. Also, we obtain 


$$
\begin{aligned}
\Psi_{\sigma, k, l} & \subset \Psi_{\sigma, k+1, l+1}, \quad(\forall k, l \in \mathbb{N}), \\
\Omega & =\bigcup_{k=1}^{\infty} \bigcup_{l=1}^{\infty} \Psi_{\sigma, k, l} .
\end{aligned}
$$

As a result, $\mu(\Omega)=\lim _{m, n \rightarrow \infty} \mu\left(\Psi_{\sigma, k, l}\right)$. Take $\varepsilon>0$. For each $k, l \in \mathbb{N}$, select $k(\sigma), l(\sigma) \in \mathbb{N}$ such that $\mu(\Omega \backslash$ $\left.\Psi_{\sigma, k(\sigma), l(\sigma)}\right)<\left(\varepsilon / 2^{\sigma}\right)$. Set

$$
T_{0}=\bigcup_{\sigma=1}^{\infty}\left(\Omega \backslash \Psi_{\sigma, k(\sigma), l(\sigma)}\right) .
$$

Then, we have

$$
\mu\left(T_{0}\right) \leq \sum_{\sigma=1}^{\infty} \mu\left(\Omega \backslash \Psi_{\sigma, k(\sigma), l(\sigma)}\right)<\varepsilon .
$$

Let

$$
T=\Omega \backslash T_{0}=\bigcap_{\sigma=1}^{\infty} \Psi_{\sigma, k(\sigma), l(\sigma)} .
$$

Thus, $\mu(\Omega \backslash T)=\mu\left(T_{0}\right)<\varepsilon$. Hence, we get $\forall \sigma \in \mathbb{N}, \forall r \geq$ $k(\sigma), u \geq l(\sigma), \forall z \in T$,

$$
\delta_{\theta_{2}}^{r u}\left(\left\{(m, n) \in \mathbb{N} \times \mathbb{N}: D\left(\bar{h}_{m n}(z), \bar{h}(z)\right) \geq \frac{1}{\sigma}\right\}\right)<\frac{1}{\sigma} .
$$

This gives that $\bar{h}_{m n \mid \mathscr{A}} \stackrel{e S_{\theta_{2}}}{\longrightarrow} \bar{h}_{\mid \mathscr{A}}$ on $\mathscr{A}$.

Now, we give the concepts of inner and outer lacunary statistical convergence in measure of DSFVF and demonstrate the equivalence of these concepts. For our convenience, we use FVMF and DSFVMF in place of fuzzy-valued function and double sequence of fuzzy-valued measurable function, respectively.

Definition 5. Take measurable space $(\Omega, \mathscr{M}, \mu)$. Presume that the set $\mathscr{L}^{0}$ of all FVMF defined almost everywhere on $\Omega$. Take $\left(\bar{h}_{m n}\right)$ and $\bar{h}$ in $\mathscr{L}^{0}$. The outer lacunary statistical convergence in measure of a DSFVMF $\left(\bar{h}_{m n}\right)$ to a FVMF $\bar{h}$ is defined by

$$
\delta_{\theta_{2}}^{r u}\left(\left\{(m, n) \in \mathbb{N} \times \mathbb{N}: \mu\left(\left\{z \in \Omega: D\left(\bar{h}_{m n}(z), \bar{h}(z)\right) \geq \eta\right\}\right) \geq \zeta\right\}\right) \longrightarrow 0, \quad \text { if } r, u \longrightarrow \infty,
$$

for $\eta, \zeta>0$. It is denoted by $\bar{h}_{m n} \longrightarrow{ }^{\delta_{\theta_{2}}}, \mu \bar{h}$. If we take (68) as follows, we get the inner statistical convergence in measure of a DSFVMF $\left(\bar{h}_{m n}\right)$ to a FVMF $\bar{h}$ :

$$
\mu\left(\left\{z \in \Omega: \delta_{\theta_{2}}^{r u}\left(\left\{(m, n) \in \mathbb{N} \times \mathbb{N}: D\left(\bar{h}_{m n}(z), \bar{h}(z)\right) \geq \eta\right\}\right) \geq \zeta\right\}\right) \longrightarrow 0, \quad \text { if } r, u \longrightarrow \infty .
$$

It is demonstrated by $\bar{h}_{m n} \longrightarrow^{\mu}, \delta_{\theta_{2}} \bar{h}$.

Theorem 6. Take measurable space $(\Omega, \mathscr{M}, \mu)$. Presume $\left(\bar{h}_{m n}\right)$ and $\bar{h}$ in $\mathscr{L}^{0}$ :

(i) If $\bar{h}_{m n} \longrightarrow{ }^{\delta_{\theta_{2}}}, \mu \bar{h}$, then $\bar{h}_{m n} \longrightarrow^{\mu}, \delta_{\theta_{2}} \bar{h}$

(ii) If $\bar{h}_{m n} \longrightarrow^{\mu}, \delta_{\theta_{2}} \bar{h}$, then $\bar{h}_{m n} \longrightarrow^{\delta_{\theta_{2}}}, \mu \bar{h}$, provided $\mu(\Omega)<\infty$

Proof. Since $\delta_{\theta_{2}}^{r u}: P_{1} \longrightarrow[0,1](r, u \in \mathbb{N})$ is a probability measure, we can think the product measure $\mu \times \delta_{\theta_{2}}^{r u}$ on the product algebra $\mathscr{M} \otimes P_{1}$ of subsets of $\Omega \times(\mathbb{N} \times \mathbb{N})$. For fix $\eta>0$, we obtain

$$
S_{\eta}=\left\{(z,(m, n)) \in \Omega \times(\mathbb{N} \times \mathbb{N}): D\left(\bar{h}_{m n}(z), \bar{h}(z)\right) \geq \eta\right\} .
$$

Define a function $\Phi: \Omega \times(\mathbb{N} \times \mathbb{N}) \longrightarrow \mathbb{R}$ as

$$
\Phi((z,(m, n)))=D\left(\bar{h}_{m n}(z), \bar{h}(z)\right),(z,(m, n)) \in \Omega \times(\mathbb{N} \times \mathbb{N}),
$$

is $\mathscr{M} \otimes P_{1}$-measurable. Therefore, we get $S_{\eta} \in \mathscr{M} \otimes P_{1}$. For any $\mathscr{K} \subset \Omega \times(\mathbb{N} \times \mathbb{N})$, one writes
$\mathscr{K}(z)=\{(m, n) \in \mathbb{N} \times \mathbb{N}:(z,(m, n)) \in \mathscr{K}\}, \quad$ if $z \in \Omega$, $\mathscr{K}(m, n)=\{z \in \Omega:(z,(m, n)) \in \mathscr{K}\}, \quad$ if $(m, n) \in(\mathbb{N} \times \mathbb{N})$.

(i) In order to acquire this, we have to show that

$$
\begin{aligned}
& \forall \varepsilon, q>0, \exists r_{0}, u_{0} \in \mathbb{N}, \forall r \\
& \quad \geq r_{0}, u \geq u_{0}, \mu\left(\left\{z \in \Omega: \delta_{\theta_{2}}^{r u}\left(S_{\eta}(z)\right) \geq q\right\}\right)<\varepsilon .
\end{aligned}
$$

Fix $q>0$ and $\varepsilon>0$. Since $\bar{h}_{m n} \longrightarrow \delta_{\theta_{2}}, \mu \bar{h}$, one can find $r_{0}, u_{0} \in \mathbb{N}$ such that $r \geq r_{0}, u \geq u_{0}$, one get the following:

$\delta_{\theta_{2}}^{r u}\left(\left\{(m, n) \in \mathbb{N} \times \mathbb{N}: \mu\left(S_{\eta}(m, n)\right) \geq 1\right\}\right)<\frac{q}{2}$,

$\delta_{\theta_{2}}^{r u}\left(\left\{(m, n) \in \mathbb{N} \times \mathbb{N}: \mu\left(S_{\eta}(m, n)\right) \geq \frac{q \varepsilon}{4}\right\}\right)<\frac{q \varepsilon}{4}$.

Assume that

$$
P=\left\{(m, n) \in \mathbb{N} \times \mathbb{N}: \mu\left(S_{\eta}(m, n)\right)<1\right\} .
$$

Then, we have from situation (74) that

$$
\delta_{\theta_{2}}^{r u}(\mathbb{N} \times \mathbb{N} \backslash P)<\frac{q}{2}\left(\forall r \geq r_{0}, u \geq u_{0}\right) .
$$


Hence, for all $\forall r \geq r_{0}$ and $u \geq u_{0}$, one acquires

$$
\begin{aligned}
& \mu\left(\left\{z \in \Omega: \delta_{\theta_{2}}^{r u}\left(S_{\eta}(z)\right) \geq q\right\}\right) \\
& \leq \mu\left(\left\{z \in \Omega: \delta_{\theta_{2}}^{r u}\left(S_{\eta}(z) \cap P\right) \geq \frac{q}{2}\right\}\right) \\
& \quad+\mu\left(\left\{z \in \Omega: \delta_{\theta_{2}}^{r u}\left(S_{\eta}(z) \backslash P\right) \geq \frac{q}{2}\right\}\right) \\
& \leq \mu\left(\left\{z \in \Omega: \delta_{\theta_{2}}^{r u}\left(S_{\eta}(z) \cap P\right) \geq \frac{q}{2}\right\}\right) .
\end{aligned}
$$

Take $S_{\eta}^{*}=S_{\eta} \cap(\Omega \times P)$. Therefore, we have

$$
\begin{aligned}
S_{\eta}^{*}(z) & =S_{\eta}(z) \cap P(z \in \Omega), \\
S_{\eta}^{*}(m, n) & =S_{\eta}(m, n)((m, n) \in P) .
\end{aligned}
$$

To acquire relation (73), it is enough to demonstrate that

$\forall r \geq r_{0}, u \geq u_{0}, \mu\left(\left\{z \in \Omega: \delta_{\theta_{2}}^{r u}\left(S_{\eta}^{*}(z)\right) \geq \frac{q}{2}\right\}\right)<\varepsilon$.

For the set $S_{\eta}^{*} \subset \Omega \times P$ and for every fix $r, u \in \mathbb{N}$, we can utilize the Fubini theorem for the characteristic function of $S_{\eta}^{*}$ of the finite measure $\mu \times \delta_{\theta_{2}}^{r u}$. Actually,

$$
S_{\eta}^{*}=\bigcup_{(m, n) \in P}\left((m, n) \times S_{\eta}(m, n)\right),
$$

where

$$
\begin{gathered}
\mu\left(S_{\eta}(m, n)\right)<1(\forall(m, n) \in P), \\
\delta_{\theta_{2}}^{r u}(\{(m, n)\})=0(\forall m>r, n>u) .
\end{gathered}
$$

Thus,

$$
\begin{aligned}
\iint_{P} \mu\left(S_{\eta}^{*}(m, n)\right) \mathrm{d} m \mathrm{~d} n & =\left(\mu \times \delta_{\theta_{2}}^{r u}\right)\left(S_{\eta}^{*}\right) \\
& =\int_{\Omega} \delta_{\theta_{2}}^{r u}\left(S_{\eta}^{*}(z)\right) \mathrm{d} z .
\end{aligned}
$$

Suppose $r_{0}$ and $u_{0} \in \mathbb{N}$ such that $r \geq r_{0}$ and $u \geq u_{0}$, one obtains

$$
\begin{aligned}
\frac{q \varepsilon}{2}> & \frac{q \varepsilon}{4}+\delta_{\theta_{2}}^{r u}\left(\left\{(m, n) \in \mathbb{N} \times \mathbb{N}: \mu\left(S_{\eta}(m, n)\right) \geq \frac{q \varepsilon}{4}\right\}\right) \\
\geq & \iint_{\left\{(m, n) \in P: \mu\left(S_{\eta}(m, n)\right)<(q \varepsilon / 4)\right\}} \mu\left(S_{q}(m, n)\right) \mathrm{d} m \mathrm{~d} n \\
& +\iint_{\left\{(m, n) \in P: \mu\left(S_{\eta}(m, n)\right) \geq(q \varepsilon / 4)\right\}} 1 \cdot \mathrm{d} m \mathrm{~d} n \\
\geq & \iint_{P} \mu\left(S_{\eta}(m, n)\right) \mathrm{d} m \mathrm{~d} n=\iint_{P} \mu\left(S_{\eta}^{*}(m, n)\right) \mathrm{d} m \mathrm{~d} n \\
= & \int_{\Omega} \delta_{\theta_{2}}^{r u}\left(S_{\eta}^{*}(z)\right) \mathrm{d} z \\
\geq & \left.\int_{\{z \in \Omega: \mu}\left(S_{\eta}^{*}(z)\right) \geq(q / 2)\right\} \\
\geq & \frac{q}{2} \mu\left(\left\{z \in \Omega: \delta_{\theta_{2}}^{r u}\left(S_{\eta}^{*}(z)\right) \geq \frac{q}{2}\right\}\right),
\end{aligned}
$$

which gives that strict inequality (80) is valid.

(ii) Presume that $\mu(\Omega)<\infty$. Fix $\eta>0$. To verify our result, we need to denote that

$$
\begin{aligned}
& \forall \mathcal{E}, q>0, \exists r_{0}, u_{0} \in \mathbb{N}, \forall r \\
& \quad \geq r_{0}, u \geq u_{0}, \delta_{\theta_{2}}^{r u}\left(\left\{(m, n) \in \mathbb{N} \times \mathbb{N}: \mu\left(S_{\eta}(m, n)\right) \geq q\right\}\right)<\varepsilon .
\end{aligned}
$$

Let $\varepsilon>0$ and $q>0$ be given. Since $\bar{h}_{m n} \longrightarrow^{\mu}, \delta_{\theta_{2}} \bar{h}$, one can find $r_{0}, u_{0} \in \mathbb{N}$ such that, for all $r \geq r_{0}$ and $u \geq u_{0}$, we have

$$
\mu\left(\left\{z \in \Omega: \delta_{\theta_{2}}^{r u}\left(S_{\eta}(z)\right) \geq \frac{q \varepsilon}{2 \mu(\Omega)}\right\}\right)<\frac{q \varepsilon}{2} .
$$

By considering the Fubini theorem for the function of $S_{\eta} \subset \Omega \times(\mathbb{N} \times \mathbb{N})$, we obtain

$$
\int_{\Omega} \delta_{\theta_{2}}^{r u}\left(S_{\eta}(z)\right) \mathrm{d} z=\left(\mu \times \delta_{\theta_{2}}^{r u}\right)\left(S_{\eta}\right)=\iint_{\mathbb{N} \times \mathbb{N}} \mu\left(S_{\eta}(m, n)\right) \mathrm{d} m \mathrm{~d} n .
$$

Supposing $r_{0}$ and $u_{0}$ such that, for all $r \geq r_{0}$ and $u \geq u_{0}$, we have

$$
\begin{aligned}
q \varepsilon & >\frac{q \varepsilon \mu(\Omega)}{2 \mu(\Omega)}+\mu\left(\left\{z \in \Omega: \delta_{\theta_{2}}^{r u}\left(S_{\eta}(z)\right) \geq \frac{q \varepsilon}{2 \mu(\Omega)}\right\}\right) \\
& \geq \int_{\left\{z \in \Omega: \delta_{\theta_{2}}^{r u}\left(S_{\eta}(z)\right)<((q \varepsilon) /(2 \mu(\Omega)))\right\}} \delta_{\theta_{2}}^{r u}\left(S_{\eta}(z)\right) \mathrm{d} z \\
& +\int_{\left\{z \in \Omega: \delta_{\theta_{2}}^{r u}\left(S_{\eta}(z)\right)>((q \varepsilon) /(2 \mu(\Omega)))\right\}} 1 \mathrm{~d} z \\
& \geq \int_{\Omega} \delta_{\theta_{2}}^{r u}\left(S_{\eta}(z)\right) \mathrm{d} z=\iint_{\mathbb{N} \times \mathbb{N}} \mu\left(S_{\eta}(m, n)\right) \mathrm{d} m \mathrm{~d} n \\
& \geq \iint_{\left\{(m, n) \in \mathbb{N} \times \mathbb{N}: \mu\left(S_{\eta}(m, n)\right) \geq q\right\}} \mu\left(S_{\eta}(m, n)\right) \mathrm{d} m \mathrm{~d} n \\
& \geq q \delta_{\theta_{2}}^{r u}\left(\left\{(m, n) \in \mathbb{N} \times \mathbb{N}: \mu\left(S_{\eta}(m, n)\right) \geq q\right\}\right) .
\end{aligned}
$$

This concludes the proof.

Theorem 6 indicates that both kinds of convergence (in Definition 5) in measure are equivalent if $\Omega$ is a finite measurable set. By thinking the set $\Omega$, we give the following definition.

Definition 6. DSFVMF $\left(\bar{h}_{m n}\right)$ is called to be lacunary statistical convergent in measure (shortly, LSCM) to a FVMF $\bar{h}$, in symbol, $\bar{h}_{m n} \longrightarrow^{\mu} S_{\theta_{2}} \bar{h}$, if $\mu\left(\left\{z \in \Omega: D\left(\bar{h}_{m n}(z)\right), \bar{h}(z)\right\} \geq\right.$ $q)$ is lacunary statistically convergent to zero for any $q>0$ and all $(m, n) \in \mathbb{N} \times \mathbb{N}$. This concept can be denoted by the following formula:

$$
\begin{aligned}
& \forall \eta>0, \forall q>0,\{(m, n) \in \mathbb{N} \times \mathbb{N}: \mu \\
& \left.\quad \cdot\left(\left\{z \in \Omega: D\left(\bar{h}_{m n}(z), \bar{h}(z)\right) \geq q\right\}\right)>\eta\right\} \in \Lambda_{2} .
\end{aligned}
$$

Here, we can take $\eta=q$ or $q=(1 / r), r \in \mathbb{N}$. 
Proposition 1. Take measurable space $(\Omega, \mathscr{M}, \mu)$. Suppose that $\left(\bar{h}_{m n}\right)$ and $\bar{h}$ in $\mathscr{L}^{0}$. Then, $\bar{h}_{m n} \stackrel{u S_{\theta_{2}}}{\rightrightarrows} \bar{h} \Rightarrow \bar{h}_{m n} \longrightarrow{ }^{\mu} S_{\theta_{2}} \bar{h}$.

Proof. We presume that $\bar{h}_{m n} \stackrel{u S_{\theta_{2}}}{\rightrightarrows} \bar{h}$. Take $q>0$. In this way, there is a set $W \in \Lambda_{2}$ such that

$$
D\left(\bar{h}_{m n}(z), \bar{h}(z)\right)<q, \quad \forall(m, n) \notin W, z \in \Omega .
$$

Thus, we obtain

$$
\begin{aligned}
& \left\{(m, n) \in \mathbb{N} \times \mathbb{N}: \mu\left(\left\{z \in \Omega: D\left(\bar{h}_{m n}(z), \bar{h}(z)\right) \geq q\right\}\right)>q\right\} \\
& \quad \subset\left\{(m, n) \in \mathbb{N} \times \mathbb{N}: \mu\left(\left\{z \in \Omega: D\left(\bar{h}_{m n}(z), \bar{h}(z)\right) \geq q\right\}\right) \neq \varnothing\right\} \\
& \quad \subset W \in \Lambda_{2} .
\end{aligned}
$$

This indicates that $\bar{h}_{m n}(z) \longrightarrow^{\mu} S_{\theta_{2}} \bar{h}(z)$.

Theorem 7. Let $\bar{h}$ be a FVMF such that, for each $z \in \Omega$, $\bar{h}_{m n}(z) \longrightarrow P S_{\theta_{2}} \bar{h}(z)$. Then, given $\varepsilon>0$ and $\delta>0$, there is a measurable set $W \subset \Omega$ with $\mu(W)<\delta$ and integers $m_{0}$ and $n_{0}$ such that

$$
D\left(\bar{h}_{m n}(z), \bar{h}(z)\right)<\varepsilon, \quad\left(\forall z \notin W \text { and } m \geq m_{0}, n \geq n_{0}\right) .
$$

Proof. Let

$$
Z_{m n}=\left\{z \in \Omega: D\left(\bar{h}_{m n}(z), \bar{h}(z)\right) \geq \varepsilon\right\},
$$

and set

$$
\begin{aligned}
\Omega_{m_{0}, n_{0}} & =\bigcup_{m=m_{0}}^{\infty} \bigcup_{n=n_{0}}^{\infty} Z_{m n} \\
& =\left\{z \in \Omega: D\left(\bar{h}_{m n}(z), \bar{h}(z)\right) \geq \varepsilon, \text { for some } m \geq m_{0}, n \geq n_{0}\right\} .
\end{aligned}
$$

We have that $\Omega_{m_{0}+1, n_{0}+1} \subset \Omega_{m_{0}, n_{0}}$, and for each $z \in \Omega$, there is some $\Omega_{m_{0}, n_{0}}$ to which $z$ does not belong, since $\bar{h}_{m n}(z) \longrightarrow \bar{h}(z)$. Thus, we get $\cap_{m_{0}} \cap_{n_{0}} \Omega_{m_{0}, n_{0}}=\varnothing$, so we have $\lim \mu\left(\Omega_{m_{0}, n_{0}}\right)=0$. Hence, given $\delta>0, \exists m_{0}, n_{0} \in \mathbb{N}$ so that $\mu\left(\Omega_{m_{0}, n_{0}}\right)<\delta$, i.e.,

$\mu\left(\left\{z \in \Omega: D\left(\bar{h}_{m n}(z), \bar{h}(z)\right) \geq \varepsilon\right.\right.$, for some $\left.\left.m \geq m_{0}, n \geq n_{0}\right\}\right)<\delta$.

If we write $W$ for this $\Omega_{m_{0}, n_{0}}$, then $\mu(W)<\delta$ and $W=\left\{z \in \Omega: D\left(\bar{h}_{m n}(z), \bar{h}(z)\right) \geq \varepsilon\right.$, for some $\left.m \geq m_{0}, n \geq n_{0}\right\}$.

Theorem 8. If DSFVMF $\left(\bar{h}_{m n}\right)$ pointwise lacunary statistically convergent to a FVMF $\bar{h}$ almost everywhere on $\Omega$, then $\bar{h}_{m n} \longrightarrow{ }^{\mu} S_{\theta_{2}} \bar{h}$.

Proof. Assume that $\bar{h}_{m n}(z) \longrightarrow^{p} S_{\theta_{2}} \bar{h}(z)$ almost everywhere on $\Omega$. We have from Theorem 6 that $\bar{h}_{m n} \longrightarrow^{\mu}, \delta_{\theta_{2}} \bar{h}$ is the same as $\bar{h}_{m n}(z) \longrightarrow^{\mu} S_{\theta_{2}} \bar{h}(z)$. So, to demonstrate our result, we have to show that $\bar{h}_{m n}(z) \longrightarrow^{\mu}, \delta_{\theta_{2}} \bar{h}(z)$. Presume that $\varepsilon>0$ and $q>0$. It is clear from Theorem 5 that $\mathscr{A} \subset \mathscr{M}$ such that $\bar{h}_{m n \mid \mathscr{A}} \longrightarrow{ }^{e} S_{\theta_{2}} \bar{h}_{\mid \mathscr{A}}$ and $\mu(\Omega \backslash \mathscr{A})<\varepsilon$. Select indexes $k$ and $l$ such that

$$
\delta_{\theta_{2}}^{r u}\left(\left\{(m, n) \in \mathbb{N} \times \mathbb{N}: D\left(\bar{h}_{m n}(z), \bar{h}(z)\right) \geq q\right\}\right)<q,
$$

for every $r \geq k, u \geq l$, and $y \in \mathscr{A}$. Thus, for all $r \geq k$ and $u \geq l$, we obtain

$$
\left\{z \in \Omega: \delta_{\theta_{2}}^{r u}\left(\left\{(m, n) \in \mathbb{N} \times \mathbb{N}: D\left(\bar{h}_{m n}(z), \bar{h}(z)\right) \geq q\right\}\right) \geq q\right\} \subset \Omega \backslash \mathscr{A} .
$$

Therefore, for every $r \geq k$ and $u \geq l$,

$\mu\left(\left\{z \in \Omega: \delta_{\theta_{2}}^{r u}\left(\left\{(m, n) \in \mathbb{N} \times \mathbb{N}: D\left(\bar{h}_{m n}(z), \bar{h}(z)\right) \geq q\right\}\right) \geq q\right\}\right)<\varepsilon$,

as desired.

Theorem 9. If $\bar{h}_{m n}(z) \longrightarrow P S_{\theta_{2}} \bar{h}(z)$ almost everywhere on $\Omega$, then $\bar{h}_{m n}(z) \longrightarrow^{\mu} S_{\theta_{2}} \bar{h}(z)$.

Proof. Take $q, \varepsilon>0$. By considering Theorem 5, there is an $\mathscr{A} \subset \mathscr{M}$ such that $\bar{h}_{m n \mid \mathscr{A}} \longrightarrow^{e} S_{\theta_{2}} \bar{h}_{\mid \mathscr{A}}$ and $\mu(\Omega \backslash \mathscr{A})<\varepsilon$. Consider $k, l \in \mathbb{N}$ such that

$$
\begin{aligned}
& \delta_{\theta_{2}}^{r u}\left(\left\{(m, n) \in \mathbb{N} \times \mathbb{N}: D\left(\bar{h}_{m n}(z), \bar{h}(z)\right) \geq q\right\}\right) \\
& \quad<q(\forall r \geq k, u \geq l \text { and } z \in \mathscr{A}),
\end{aligned}
$$

which yields

$$
\begin{aligned}
\{z & \left.\in \Omega: \delta_{\theta_{2}}^{r u}\left(\left\{(m, n) \in \mathbb{N} \times \mathbb{N}: D\left(\bar{h}_{m n}(z), \bar{h}(z)\right) \geq q\right\}\right) \geq q\right\} \\
& \subset \Omega \backslash \mathscr{A}(\forall r \geq k, u \geq l) .
\end{aligned}
$$

Therefore, one obtains

$\mu\left(\left\{z \in \Omega: \delta_{\theta_{2}}^{r u}\left(\left\{(m, n) \in \mathbb{N} \times \mathbb{N}: D\left(\bar{h}_{m n}(z), \bar{h}(z)\right) \geq q\right\}\right) \geq q\right\}\right)<\varepsilon$.

Corollary 2. If $\bar{h}_{m n}(z) \longrightarrow{ }^{\mu} S_{\theta_{2}} \bar{h}(z)$, then $\exists$ a subsequence $\left(\bar{h}_{m_{k} n_{l}}\right)$ of $\left(\bar{h}_{m n}\right)$ such that $\bar{h}_{m_{k} n_{l}}^{2}(z) \longrightarrow p S_{\theta_{2}} \bar{h}(z)$ almost everywhere on $\Omega$.

Proof. Presume that $\bar{h}_{m n}(z) \longrightarrow^{\mu} S_{\theta_{2}} \bar{h}(z)$, so any subsequence $\left(\bar{h}_{m_{k} n_{l}}\right)$ of $\left(\bar{h}_{m n}\right)$ also lacunary statistically convergent in measure to $\bar{h}$. In this way, $\left(\bar{h}_{m n}\right)$ possesses a subsequence that lacunary statistically convergent in measure to $\bar{h}$ almost everywhere on $\Omega$. This gives that $\bar{h}_{m_{k} n_{l}}(z) \longrightarrow p S_{\theta_{2}} \bar{h}(z)$ almost everywhere on $\Omega$.

Definition 7. Take finite measurable space $(\Omega, \mathscr{M}, \mu)$. Assume that $\left(\bar{h}_{m n}\right)_{\alpha}^{+},\left(\bar{h}_{m n}\right)_{\alpha}^{-}, \bar{h}_{\alpha}^{+}$, and $\bar{h}_{\alpha} \in \mathscr{L}^{0}$. The double sequence $\left[\bar{h}_{m n}(z)\right]_{\alpha}$ is uniformly lacunary statistically convergent in measure (briefly, we can write ULSCM) to $[\bar{h}(z)]_{\alpha}$ with regards to $\alpha$ if 


$$
\begin{aligned}
& \mu\left(\left\{z \in \Omega: \sup _{\alpha \in[0,1]}\left|\left(\bar{h}_{m n}(z)\right)_{\alpha}^{+}-(\bar{h}(z))_{\alpha}^{+}\right| \geq q\right\}\right), \\
& \mu\left(\left\{z \in \Omega: \sup _{\alpha \in[0,1]}\left|\left(\bar{h}_{m n}(z)\right)_{\alpha}^{-}-(\bar{h}(z))_{\alpha}^{-}\right| \geq q\right\}\right),
\end{aligned}
$$

both lacunary statistically convergent measure to zero for each $q>0$. Notice that this concept is given by the following formula:

$$
\begin{array}{r}
\forall \eta>0, \forall q>0,\left\{(m, n) \in \mathbb{N} \times \mathbb{N}: \mu\left(\left\{z \in \Omega: \sup _{\alpha \in[0,1]}\left|\left(\bar{h}_{m n}(z)\right)_{\alpha}^{+}-(\bar{h}(z))_{\alpha}^{+}\right| \geq q\right\}\right) \geq \eta\right\} \in \Lambda_{2}, \\
\forall \eta>0, \forall q>0,\left\{(m, n) \in \mathbb{N} \times \mathbb{N}: \mu\left(\left\{z \in \Omega: \sup _{\alpha \in[0,1]}\left|\left(\bar{h}_{m n}(z)\right)_{\alpha}^{-}-(\bar{h}(z))_{\alpha}^{-}\right| \geq q\right\}\right) \geq \eta\right\} \in \Lambda_{2} . \\
\mu\left(\left\{z \in \Omega: D \bar{h}_{m n}(z), \bar{h}(z) \geq t q\right\}\right),
\end{array}
$$

In that case, we can write $\eta=q$ or $q=(1 / r), r \in \mathbb{N}$.

is lacunary statistically convergent to zero for each $q>0$, i.e., $\left\{(m, n) \in \mathbb{N} \times \mathbb{N}: \mu\left(\left\{z \in \Omega: D\left(\bar{h}_{m n}(z), \bar{h}(z)\right) \geq q\right\}\right) \geq q\right\} \in \Lambda_{2}$.

Theorem 10. DSFVMF $\left(\bar{h}_{m n}\right)$ is LSCM to FVMF $\bar{h}$ iff $\left[\bar{h}_{m n}(z)\right]_{\alpha}$ is ULSCM to $[\bar{h}(z)]_{\alpha}$ with regards to $\alpha$.

Proof. Suppose that $\left(\bar{h}_{m n}\right)$ is LSCM to $\bar{h}$. Then,

Thus,

$$
\left\{(m, n) \in \mathbb{N} \times \mathbb{N}: \mu\left(\left\{z \in \Omega: \sup _{\alpha \in[0,1]} \max \left\{\left|\left(\bar{h}_{m n}(z)\right)_{\alpha}^{-}-(\bar{h}(z))_{\alpha}^{-}\right|,\left|\left(\bar{h}_{m n}(z)\right)_{\alpha}^{+}-(\bar{h}(z))_{\alpha}^{+}\right|\right\} \geq q\right\}\right) \geq q\right\} \in \Lambda_{2} .
$$

For every $q>0$, one obtains

$$
\begin{aligned}
& \left\{(m, n) \in \mathbb{N} \times \mathbb{N}: \mu\left(\left\{z \in \Omega: \sup _{\alpha \in[0,1]}\left|\left(\bar{h}_{m n}(z)\right)_{\alpha}^{-}-(\bar{h}(z))_{\alpha}^{-}\right| \geq q\right\}\right) \geq q\right\} \in \Lambda_{2}, \\
& \left\{(m, n) \in \mathbb{N} \times \mathbb{N}: \mu\left(\left\{z \in \Omega: \sup _{\alpha \in[0,1]}\left|\left(\bar{h}_{m n}(z)\right)_{\alpha}^{+}-(\bar{h}(z))_{\alpha}^{+}\right| \geq q\right\}\right) \geq q\right\} \in \Lambda_{2},
\end{aligned}
$$

which gives that

$$
\mu\left(\left\{z \in \Omega: \sup _{\alpha \in[0,1]}\left|\left(\bar{h}_{m n}(z)\right)_{\alpha}^{-}-(\bar{h}(z))_{\alpha}^{-}\right| \geq q\right\}\right) \stackrel{\mu}{\longrightarrow} S_{\theta_{2}} 0, \mu\left(\left\{z \in \Omega: \sup _{\alpha \in[0,1]}\left|\left(\bar{h}_{m n}(z)\right)_{\alpha}^{+}-(\bar{h}(z))_{\alpha}^{+}\right| \geq q\right\}\right) \stackrel{\mu}{\longrightarrow} S_{\theta_{2}} 0
$$

Hence, $\left[\bar{h}_{m n}(z)\right]_{\alpha}$ is ULSCM to $[\bar{h}(z)]_{\alpha}$ with regards to $\alpha$.

Next, we presume that $\left[\bar{h}_{m n}(z)\right]_{\alpha}$ is ULSCM to $[\bar{h}(z)]_{\alpha}$ with regards to $\alpha$. Then, for every $q>0$, one has

$$
\mu\left(\left\{z \in \Omega: \sup _{\alpha \in[0,1]}\left|\left(\bar{h}_{m n}(z)\right)_{\alpha}^{-}-(\bar{h}(z))_{\alpha}^{-}\right| \geq q\right\}\right) \stackrel{\mu}{\longrightarrow} S_{\theta_{2}} 0, \mu\left(\left\{z \in \Omega: \sup _{\alpha \in[0,1]}\left|\left(\bar{h}_{m n}(z)\right)_{\alpha}^{+}-(\bar{h}(z))_{\alpha}^{+}\right| \geq q\right\}\right) \stackrel{\mu}{\longrightarrow} S_{\theta_{2}} 0 .
$$


Thus,

$$
\begin{aligned}
& \left\{(m, n) \in \mathbb{N} \times \mathbb{N}: \mu\left(\left\{z \in \Omega: \sup _{\alpha \in[0,1]}\left|\left(\bar{h}_{m n}(z)\right)_{\alpha}^{-}-(\bar{h}(z))_{\alpha}^{-}\right| \geq q\right\}\right) \geq q\right\} \in \Lambda_{2}, \\
& \left\{(m, n) \in \mathbb{N} \times \mathbb{N}: \mu\left(\left\{z \in \Omega: \sup _{\alpha \in[0,1]}\left|\left(\bar{h}_{m n}(z)\right)_{\alpha}^{+}-(\bar{h}(z))_{\alpha}^{+}\right| \geq q\right\}\right) \geq q\right\} \in \Lambda_{2} .
\end{aligned}
$$

From the last two relations, we obtain

$$
\left\{(m, n) \in \mathbb{N} \times \mathbb{N}: \mu\left(\left\{z \in \Omega: \sup _{\alpha \in[0,1]} \max \left\{\left|\left(\bar{h}_{m n}(z)\right)_{\alpha}^{-}-(\bar{h}(z))_{\alpha}^{-}\right|,\left|\left(\bar{h}_{m n}(z)\right)_{\alpha}^{+}-(\bar{h}(z))_{\alpha}^{+}\right|\right\} \geq q\right\}\right) \geq q\right\} \in \Lambda_{2}
$$

which gives that

$$
\left\{(m, n) \in \mathbb{N} \times \mathbb{N}: \mu\left(\left\{z \in \Omega: D\left(\bar{h}_{m n}(z), \bar{h}(z)\right) \geq q\right\}\right) \geq q\right\} \in \Lambda_{2} .
$$

It means that $\left(\bar{h}_{m n}\right)$ is LSCM to FVMF $\bar{h}$.

\section{Conclusion 1}

In this study, we propose the notions of pointwise lacunary statistical convergence, uniformly lacunary statistical convergence, and equi-lacunary statistical convergence of the double sequence of fuzzy-valued functions and discuss the reationship between various kinds of lacunary statistical convergence for the double sequence of fuzzyvalued functions and the sequence of $\alpha$-level cut functions. In addition, we obtain the Egorov theorem for the double sequence of fuzzy-valued functions and investigate the lacunary statistical convergence in measure for the double sequence of fuzzy-valued functions. Note that, in contrast to the case for single sequences, a convergent double sequence need not be bounded, so the studies on lacunary statistical convergence of double sequences have a rapid growth and an emerging area in mathematical research. We conclude that our results are more general than the one proved earlier for single sequences by Kişi and Dündar. As an application, researchers who linked two theories such as the theory of approximation and the theory of lacunary statistical summability may prove fuzzy analogue of Korovkin's type approximation theorem for several test functions by using our convergence methods.

\section{Data Availability}

No data were used to support this study.

\section{Conflicts of Interest}

The authors declare that they have no conflicts of interest.

\section{References}

[1] H. Fast, "Sur la convergence statistique," Colloquium Mathematicum, vol. 2, no. 3-4, pp. 241-244, 1951.

[2] J. A. Fridy, "On statistical convergence," Analysis, vol. 5, pp. 301-313, 1985.

[3] T. Salát, "On statistically convergent sequences of real numbers," Mathematica Slovaca, vol. 30, pp. 139-150, 1980.

[4] M. Mursaleen and O. H. H. Edely, "Statistical convergence of double sequences," Journal of Mathematical Analysis and Applications, vol. 288, no. 1, pp. 223-231, 2003.

[5] M. Balcerzak, K. Dems, and A. Komisarski, "Statistical convergence and ideal convergence for sequences of functions," Journal of Mathematical Analysis and Applications, vol. 328, no. 1, pp. 715-729, 2007.

[6] A. Gökhan, M. Güngör, and M. Et, "Statistical convergence of double sequences of real-valued functions," International Mathematical Forum, vol. 2, no. 5-8, pp. 365-374, 2007.

[7] O. Duman and C. Orhan, " $\mu$-Statistically convergent function sequences," Czechoslovak Mathematical Journal, vol. 54, no. 129, pp. 413-422, 2004.

[8] J. Fridy and C. Orhan, "Lacunary statistical convergence," Pacific Journal of Mathematics, vol. 160, no. 1, pp. 43-51, 1993.

[9] C. Çakan, B. Altay, and H. Çoşkun, "Double lacunary density and lacunary statistical convergence of double sequences," Studia Scientiarum Mathematicarum Hungarica, vol. 47, no. 1, pp. 35-45, 2010.

[10] L. A. Zadeh, "Fuzzy sets," Information and Control, vol. 8, no. 3, pp. 338-353, 1965.

[11] M. Matloka, "Sequences of fuzzy numbers," Busefal, vol. 28, pp. 28-37, 1986.

[12] S. Nanda, "On sequences of fuzzy numbers," Fuzzy Sets and Systems, vol. 33, no. 1, pp. 123-126, 1989.

[13] C. V. Negoita and D. Ralescu, Applications of Fuzzy Sets to Systems Analysis, Wiley, New York, NY, USA, 1975.

[14] F. Nuray and E. Savaş, "Statistical convergence of sequences of fuzzy numbers," Mathematica Slovaca, vol. 45, no. 3, pp. 269-273, 1995.

[15] E. Savaş and M. Mursaleen, "On statistically convergent double sequences of fuzzy numbers," Information Sciences, vol. 162, no. 3-4, pp. 183-192, 2004.

[16] S. Aytar and S. Pehlivan, "Statistical convergence of sequences of fuzzy numbers and sequences of $\alpha$-cuts," International Journal of General Systems, vol. 37, no. 2, pp. 231-237, 2008. 
[17] Y. Altin, M. Et, and B. C. Tripathy, "On pointwise statistical convergence sequences of fuzzy mappings," Kuwait Journal of Science, vol. 15, no. 2, pp. 425-433, 2007.

[18] H. Altinok, Y. Altin, and M. Işık, "Statistical convergence and strong $p$-Cesàro summability of order in sequences of fuzzy numbers," Iranian Journal of Fuzzy Systems, vol. 9, no. 2, pp. $65-75,2012$.

[19] H. Altinok and M. Et, "Statistical convergence of order $\beta, \gamma$ for sequences of fuzzy numbers," Soft Computing, vol. 23, no. 15, pp. 6017-6022, 2019.

[20] M. Çınar, M. Karakaş, and M. Et, "On pointwise and uniform statistical convergence of order $\alpha$ for sequences of functions," Fixed Point Theory and Applications, vol. 33, 2020.

[21] S. A. Mohiuddine, A. Asiri, and B. Hazarika, "Weighted statistical convergence through difference operator of sequences of fuzzy numbers with application to fuzzy approximation theorems," International Journal of General Systems, vol. 48, no. 5, pp. 492-506, 2019.

[22] S. A. Mohiuddine, B. Hazarika, and A. Alotaibi, "On statistical convergence of double sequences of fuzzy valued functions," Journal of Intelligent \& Fuzzy Systems, vol. 32, no. 6, pp. 4331-4342, 2017

[23] F. Nuray, "Lacunary statistical convergence of sequences of fuzzy numbers," Fuzzy Sets and Systems, vol. 99, no. 3, pp. 353-355, 1998.

[24] E. Savaş and M. Gürdal, "Certain summability methods in intuitionistic fuzzy normed spaces," Journal of Intelligent and Fuzzy Systems, vol. 27, no. 4, pp. 1621-1629, 2014.

[25] E. Savaş and M. Gürdal, "Generalized statistically convergent sequences of functions in fuzzy 2-normed spaces," Journal of Intelligent and Fuzzy Systems, vol. 27, no. 4, pp. 2067-2075, 2014.

[26] E. Savaş and M. Gürdal, "A generalized statistical convergence in intuitionistic fuzzy normed spaces," Science Asia, vol. 41, pp. 289-294, 2015.

[27] E. Savaş, "A note on double sequences of fuzzy numbers," Turkish Journal of Mathematics, vol. 20, no. 2, pp. 175-178, 1996.

[28] E. Savaş, "On statistically convergent sequences of fuzzy numbers," Information Sciences, vol. 137, no. 1-4, pp. 277282, 2001.

[29] C. Şençimen and S. Pehlivan, "Statistical convergence in fuzzy normed linear spaces," Fuzzy Sets and Systems, vol. 159, pp. 361-370, 2008.

[30] Z. Gong, L. Zhang, and X. Zhu, "The statistical convergence for sequences of fuzzy-number-valued functions," Information Sciences, vol. 295, pp. 182-195, 2015.

[31] B. Hazarika, A. Alotaibi, and S. A. Mohiuddine, "Statistical convergence in measure for double sequences of fuzzy-valued functions," Soft Computing, vol. 24, no. 9, pp. 6613-6622, 2020.

[32] Ö. Kişi and E. Dündar, "Lacunary statistical convergence in measure for sequences of fuzzy valued functions," Konuralp Journal of Mathematics, vol. 8, no. 2, pp. 252-262, 2020. 